\title{
A REVIEW OF THE DECOHERENT HISTORIES APPROACH TO QUANTUM MECHANICS
}

\author{
J. J. HALLiWELL \\ Theory Group, Blackett Laboratory, Imperial College \\ London, SW7 2BZ, United Kingdom
}

\begin{abstract}
I review the decoherent (or consistent) histories approach to quantum mechanics, due to Griffiths, to Gell-Mann and Hartle, and to Omnès. This is an approach to standard quantum theory specifically designed to apply to genuinely closed systems, up to and including the entire universe. It does not depend on an assumed separation of classical and quantum domains, on notions of measurement, or on collapse of the wave function. Its primary aim is to find sets of histories for closed systems exhibiting negligble interference, and therefore, to which probabilities may be assigned. Such sets of histories are called consistent or decoherent, and may be manipulated according to the rules of ordinary (Boolean) logic. The approach provides a framework from which one may discuss the emergence of an approximately classical domain for macroscopic systems, together with the conventional Copenhagen quantum mechanics for microscropic subsystems. In the special case in which the total closed system naturally separates into a distinguished subsystem coupled to an environment, the decoherent histories approach is closed related to the quantum state diffusion approach of Gisin and Percival.
\end{abstract}

(To appear in proceedings of the conference,

Fundamental Problems in Quantum Theory, Baltimore, June 18-22, 1994, edited by D.Greenberger)

Imperial College preprint IC/93-94/52. July 1994

\section{Introduction}

Quantum mechanics was originally developed to account for a number of unexplained phenomena on the atomic scale. The theory was not thought to be applicable to physics at larger scales, nor was their felt any need to do so. Indeed, it was only by reference to an external, classical, macroscopic world that the theory could be properly understood. This view of quantum mechanics, the Copenhagen interpretation, has persisted for a very long time with not one shred of experimental evidence against it [1]. 
Today, however, more ambitious views of quantum mechanics are entertained.

Experiments have been contemplated (e.g., involving SQUIDS) that may probe domains traditionally thought of as macroscopic [2]. Even in the absence of such experiments, the Copenhagen interpretation rests on unsatisfactory foundations. Macrosopic classical objects are made from microscopic quantum ones. The dualist view of the Copenhagen interpretation may therefore be internally inconsistent, and is at best approximate. Most significantly, there has been a considerable amount of recent interest in the subject of quantum cosmology in which the notion of an external classical domain is completely inappropriate [3]. Generalizations of conventional quantum theory are required to meet these new challenges.

John Wheeler was one of the very first people to be so bold as to even talk about "the wave function of the universe" [4]. He has contributed extensively to our understanding of quantum mechanics and quantum cosmology, both through his own work, and through his inspiration of many others in the field. It is a great pleasure to contribute to this meeting organized in his honour.

\subsection{The Histories Approach}

The object of this paper is to review one particular approach to quantum mechanics that was specifically designed to overcome some of the problems of the orthodox approach. This is the decoherent (or "consistent") histories approch due to Griffiths [5,6,7,8,9], Gell-Mann and Hartle [10,11,12,13,14,15,16,17,18,19] and Omnès $[20,21,22,23,24,25,26]$. It is, in particular, a predictive formulation of quantum mechanics for genuninely closed quantum systems that is sufficiently general to cope with the needs of quantum cosmology. In brief, its aims are as follows:

1. To understand the emergence of an approximately classical universe from an underlying quantum one, without becoming embroiled in the details of observers, measuring devices or collapse of the wave function. Prediction of a classical domain similar to the one in which we live will generally depend on the initial condition of the universe, and moreover, could be one of many possibilities predicted by quantum mechanics. Accommodation, rather than absolute prediction, of our particular classical universe may be as much as can be expected.

2. To supply a quantum-mechanical framework for reasoning about the properties of closed physical systems. Such a framework is necessary if the process of pre- 
diction in quantum mechanics is to be genuinely quantum-mechanical at every single step. That process consists of first logically reconstructing the past history of the universe from records existing in the classical domain at the present, and then using the present records together with the deduced past history to make predictions about the future (strictly speaking, about correlations between records at a fixed moment of time in the future). A framework for reasoning may also lead to clarification of many of the conceptually troublesome aspects of quantum mechanics, such as the EPR paradox.

In more detail, the primary mathematical aim of the histories approach is to assign probabilities to histories of a closed system. The approach is a modest generalization of ordinary quantum mechanics, but relies on a far smaller list of axioms. These axioms are basically the statements that the closed system is described by the usual mathematical machinery of Hilbert together with a formula for the probabilities of histories and a rule of interpretation. It makes no distinction between microscopic and macroscopic, nor does it assume a "system-environment" split; in particular, a separate classical domain is not assumed. It makes no essential use of measurement, or collapse of the wave function, although these notions may be discussed within the framework of the approach. What replaces measurement is the more general and objective notion of consistency (or the stronger notion of decoherence), determining which histories may be assigned probabilities. The approach also stresses classical (i.e. Boolean) logic, the conditions under which it may be applied, and thus, the conditions under which ordinary reasoning may be applied to physical system.

The decoherent histories approach is not designed to answer the question held by some to be the most important problem of quantum measurement theory: why one particular history for the universe "actually happens" whilst the other potential histories allowed by quantum mechanics fade away. Although some aspects of this problem are clarified by the decoherent histories approach, a satisfactory solution does not appear to be possible unless something external is added (see Ref.[27], for example). Nor is the approach intended to meet some philosophical prejudice about the way the world appears to be. Its aims are for the large part of a rather pragmatic nature, namely answering the very physical question of why the world is described so well by classical mechanics and ordinary logic, when its atomic constituents are 


\subsection{Why histories?}

The basic building blocks in the decoherent histories approach are the histories of a closed system - sequences of alternatives at a succession of times. Why are these objects of particular interest?

(a) Histories are the most general class of situations one might be interested in. In a typical experiment, for example, a particle is emitted from a decaying nucleus at time $t_{1}$, then it passes through a magnetic field at time $t_{2}$, then it is absorbed by a detector at time $t_{3}$.

(b) We would like to understand how classical behaviour can emerge from the quantum mechanics of closed systems. This involves showing, amongst other things, that successive positions in time of a particle, say, are approximately correlated according to classical laws. This involves the probabilities for approximate positions at different times.

(c) The basic pragmatic aim of theoretical physics is to find patterns in presently existing data. In cosmology, for example, one tries to explain the connections between observed data about the microwave background, the expansion of the universe, the distribution of matter in the universe, the spectrum of gravitational waves, etc. Why, then, should we not attempt to formulate our theories in the terms of the density matrix of the entire universe at the present moment? There are at least two reasons why not. First, present records are stored in a wide variety of different ways - in computer memories, on photographic plates, on paper, in our own personal memories, in measuring devices. The dynamical variables describing those records could be very hard to identify. The correlations between present records are far easier to understand in terms of histories. The patterns in current cosmological data, for example, are explained most economically by appealing to the big bang model of the history of the universe. Second, the correlation between present records and past events can never be perfect. In order to discuss the approximate nature of correlations between the past and the present it becomes necessary to talk about the histories of a 


\section{The Formalism of Decoherent Histories}

I now briefly outline the mathematical formalism of the decoherent histories approach. Further details may be found in the original papers cited above.

\subsection{Probabilities for Histories}

In quantum mechanics, propositions about the attributes of a system at a fixed moment of time are represented by sets of projections operators. The projection operators $P_{\alpha}$ effect a partition of the possible alternatives $\alpha$ a system may exhibit at each moment of time. They are exhaustive and exclusive,

$$
\sum_{\alpha} P_{\alpha}=1, \quad P_{\alpha} P_{\beta}=\delta_{\alpha \beta} P_{\alpha}
$$

A projector is said to be fine-grained if it is of the form $|\alpha\rangle\langle\alpha|$, where $\{|\alpha\rangle\}$ are a complete set of states; otherwise it is coarse-grained. A quantum-mechanical history is characterized by a string of time-dependent projections, $P_{\alpha_{1}}^{1}\left(t_{1}\right), \cdots P_{\alpha_{n}}^{n}\left(t_{n}\right)$, together with an initial state $\rho$. The time-dependent projections are related to the time-independent ones by

$$
P_{\alpha_{k}}^{k}\left(t_{k}\right)=e^{i H\left(t_{k}-t_{0}\right)} P_{\alpha_{k}}^{k} e^{-i H\left(t_{k}-t_{0}\right)}
$$

where $H$ is the Hamiltonian. The candidate probability for such histories is

$$
p\left(\alpha_{1}, \alpha_{2}, \cdots \alpha_{n}\right)=\operatorname{Tr}\left(P_{\alpha_{n}}^{n}\left(t_{n}\right) \cdots P_{\alpha_{1}}^{1}\left(t_{1}\right) \rho P_{\alpha_{1}}^{1}\left(t_{1}\right) \cdots P_{\alpha_{n}}^{n}\left(t_{n}\right)\right)
$$

This expression is a familiar one from quantum measurement theory, but the interpretation is different. Here it is the probability for a sequence of alternatives for a closed system. The alternatives at each moment of time are characterized by projectors. The projectors are generally not associated with measurements, as they would be in the Copenhagen view of the formula (2.3). They cannot be because the system is closed.

It is straightforward to show that (2.3) is both non-negative and normalized to unity when summed over $\alpha_{1}, \cdots \alpha_{n}$. However, (2.3) does not satisfy all the axioms 
of probability theory, and for that reason it is referred to as a candidate probability. It does not satisfy the requirement of additivity on disjoint regions of sample space. More precisely, for each set of histories, one may construct coarser-grained histories by grouping the histories together. This may be achieved, for example, by summing over the projections at each moment of time,

$$
\bar{P}_{\bar{\alpha}}=\sum_{\alpha \in \bar{\alpha}} P_{\alpha}
$$

(although this is not the most general type of coarse graining). The additivity requirement is then that the probabilities for each coarser-grained history should be the sum of the probabilities of the finer-grained histories of which it is comprised. Quantum-mechanical interference generally prevents this requirement from being satisfied; thus histories of closed quantum systems cannot in general be assigned probabilities.

The standard illustrative example is the double slit experiment. The histories consist of projections at two moments of time: projections determining which slit the particle went through at time $t_{1}$, and projections determing the point at which the particle hit the screen at time $t_{2}$. As is well-known, the probability distribution for the interference pattern on the screen cannot be written as a sum of the probabilities for going through each slit; hence the candidate probabilities do not satisfy the additivity requirement.

There are, however, certain types of histories for which interference is negligible, and the candidate probabilities for histories do satisfy the sum rules. These histories may be found using the decoherence functional:

$$
D\left(\underline{\alpha}, \underline{\alpha}^{\prime}\right)=\operatorname{Tr}\left(P_{\alpha_{n}}^{n}\left(t_{n}\right) \cdots P_{\alpha_{1}}^{1}\left(t_{1}\right) \rho P_{\alpha_{1}^{\prime}}^{1}\left(t_{1}\right) \cdots P_{\alpha_{n}^{\prime}}^{n}\left(t_{n}\right)\right)
$$

Here $\underline{\alpha}$ denotes the string $\alpha_{1}, \alpha_{2}, \cdots \alpha_{n}$. Intuitively, the decoherence functional measures the amount of interference between pairs of histories. It may be shown that the additivity requirement is satisfied for all coarse-grainings if and only if

$$
\operatorname{Re} D\left(\underline{\alpha}, \underline{\alpha}^{\prime}\right)=0
$$

for all distinct pairs of histories $\underline{\alpha}, \underline{\alpha}^{\prime}[5]$. Such sets of histories are said to be consistent, or weakly decoherent. (Note that this definition of consistency is stronger 
than that originally introduced by Griffiths [5]. See Ref.[12] for a discussion of this point).

\subsection{Consistency and Classical Logic}

Why are sets of consistent histories are of interest? As stated, propositions about the attributes of a quantum system may be represented by projection operators. The set of all projections have the mathematical structure of a lattice. This lattice is non-distributive, and this means that the corresponding propositions may not be submitted to Boolean logic. Similar remarks hold for the more complex propositions expressed by general sets of quantum-mechanical histories.

The reason why consistent sets of histories are of interest is that they can be submitted to Boolean logic. Indeed, a theorem of Omnès states that a set of histories forms a consistent representation of Boolean logic if and only if it is a consistent set $[20,25,26]$. That is, in a consistent set of histories, each history corresponds to a proposition about the properties of a physical system and we can meaningfully manipulate these propositions without contradiction using ordinary classical logic. It is in this sense that the decoherent histories approach supplies a foundation for reasoning about closed physical systems.

An important example is the case of retrodiction of the past from present data. Suppose we have a consistent set of histories. We would say that the alternative $\alpha_{n}$ (present data) implies the alternatives $\alpha_{n-1} \cdots \alpha_{1}$ (past events) if

$$
p\left(\alpha_{1}, \cdots \alpha_{n-1} \mid \alpha_{n}\right) \equiv \frac{p\left(\alpha_{1}, \cdots \alpha_{n}\right)}{p\left(\alpha_{n}\right)}=1
$$

In this way, we can in quantum mechanics build a picture of the history of the universe, given the present data and the initial state, using only logic and the consistency of the histories. We can meaningfully talk about the past properties of the universe even though there was no measuring device there to record them.

There is, however, a caveat. It is very frequently the case that the same initial state and present data will admit two or more inequivalent sets of consistent histories the union of which is not a consistent set. There then often exist propositions about the past properties of the system that are logically implied by the present data in some sets of histories but not in others. Omnès refers to such propositions as "reliable", whilst propositions that are implied by the present data in every 
consistent set of histories are labeled "true" [28] (see also Ref.[29]). The existence of these so-called multiple logics means that one cannot say that past properties corresponding to reliable propositions "actually happened", because they depend on a particular choice of consistent histories. In the histories approach, the reconstruction of history from present records is therefore not unique. This means that the approach does not in general allow one to talk about the past history of the universe "the way it really is".

Is this a problem? Some feel that it is [30]. For the immediate practical purposes of quantum cosmology, however, it does not appear to be a difficulty. Recall that what quantum mechanics must ultimately explain is the correlation between records at a fixed moment of time. As stated earlier, it is easiest to understand those correlations in terms of histories, but histories enter as an intermediate step. The correlations between two records at a fixed moment of time predicted by quantum mechanics are unambiguous, even though the histories corresponding to these records may not be unique.

\section{Decoherence, Correlation and Records}

How may the consistency condition (2.6) come to be satisfied? First of all, it is straightforward to show that, with some exceptions, histories of completely finegrained projection operators will generally not lead to consistency. The consistency condition is generally satisfied only by sets of histories that are coarse-grained. When sets of histories satisfy the consistency condition (2.6) as a result of coarsegraining, they typically satisfy, in addition, the stronger condition that both the real and imaginary parts of the off-diagonal terms of the decoherence functional vanish,

$$
D\left(\underline{\alpha}, \underline{\alpha}^{\prime}\right)=0, \quad \text { for } \quad \underline{\alpha} \neq \underline{\alpha}^{\prime}
$$

This I shall refer to quite simply as decoherence. (It is sometimes referred to more specifically as medium decoherence [12] but we shall not do so here).

Physically, decoherence is intimately related the existence of records about the system somewhere in the universe. In this sense decoherence replaces and generalizes the notion of measurement in ordinary quantum mechanics. Sets of histories decohere, and hence the system "acquires definite properties", not necessarily through 
measurement, but through the interactions and correlations of the variables that are followed with the variables that are ignored as a result of the coarse-graining.

Decoherence is typically only approximate so measures of approximate decoherence are required. First, note that the decoherence functional obeys the simple inequality [31],

$$
\left|D\left(\underline{\alpha}, \underline{\alpha}^{\prime}\right)\right|^{2} \leq D(\underline{\alpha}, \underline{\alpha}) D\left(\underline{\alpha}^{\prime}, \underline{\alpha}^{\prime}\right)
$$

Intuitively, this result indicates that there can be no interference with a history which has candidate probability zero. It also suggests a possible measure of approximate decoherence: we say that a system decoheres to order $\epsilon$ if the decoherence functional satisfies (3.2) with a factor of $\epsilon^{2}$ multiplying right-hand side. This condition may be shown to imply that most (but not all) probability sum rules will then be satisfied to order $\epsilon$ [31].

Approximate decoherence to order $\epsilon$ means that the probabilities are defined only up to that order. In typical cases, $\epsilon$ is substantially smaller than any other effect that could conceivably modify the probabilities, and hence they may be thought of as precisely defined for all practical purposes. Alternatively, it has been conjectured that a generic approximately decoherent set of histories may be turned into an exactly decoherent set by modifying to order $\epsilon$ the operators projected onto at each moment of time [30].

\subsection{Records Imply Decoherence}

I now exemplify the connection between records and decoherence. Consider a closed system $S$ which consists of two weakly interacting subsystems $A$ and $B$. The Hilbert space $\mathcal{H}$ of $S$ is therefore of the form $\mathcal{H}_{A} \otimes \mathcal{H}_{B}$. For simplicity let $\mathcal{H}_{A}$ and $\mathcal{H}_{B}$ have the same dimension. Suppose we are interested in the histories characterized solely by properties of system $A$, thus $B$ is regarded as the environment. The system is analyzed using the decoherence functional (2.5), where we take the $P_{\alpha}$ to denote a projection on $\mathcal{H}_{A}$ ( hence the projections in the decoherence functional are of the form $P_{\alpha} \otimes I^{B}$, where $I^{B}$ denotes the identity on $\mathcal{H}_{B}$ ). I also introduce projections $R_{\beta}$ on the Hilbert space $\mathcal{H}_{B}$.

I shall show that histories of $A$ satisfy the decoherence condition (3.1) if the sequences of alternatives the histories consist of exhibit exact and persistent corre- 
lations with sequences of alternatives of $B$. To be precise, suppose that the alternatives of $A$ characterized by $P_{\alpha_{k}}^{k}$ at each moment of time $t_{k}$ are perfectly recorded in $B$ as a result of their interaction. Suppose also that this record in $B$ is perfectly persistent (i.e., permanent). This means that at any time $t_{f}$ after the time $t_{n}$ of the last projection on $A$ there exist a sequence of alternatives of $B, \beta_{1}, \cdots \beta_{n}$, that are in perfect correlation with the alternatives of $A, \alpha_{1} \cdots \alpha_{n}$ at times $t_{1} \cdots t_{n}$.

For each moment of time $t_{k}$, the decoherence functional (2.5) may be written,

$$
D\left(\underline{\alpha}, \underline{\alpha}^{\prime}\right)=\sum_{\beta_{k}} \operatorname{Tr}\left(I^{A} \otimes R_{\beta_{k}}^{k} \cdots P_{\alpha_{k}}^{k} \otimes I^{B} \cdots \rho \cdots P_{\alpha_{k}^{\prime}}^{k} \otimes I^{B} \cdots\right)
$$

using the exhaustivity of the projections $R_{\beta_{k}}^{k}$, where the dots denote the projections at times other than $t_{k}$ and the unitary evolution operators between them. Now, since $R_{\beta_{k}}^{k}$ a projector, it may be replaced by $\left(R_{\beta_{k}}^{k}\right)^{2}$. Furthermore, the assumption of persistence then allows us to move the projector $R_{\beta_{k}}^{k}$ through all the unitary evolution operators occuring after time $t_{k}$ on each side of the decoherence functional, with the result,

$$
D\left(\underline{\alpha}, \underline{\alpha}^{\prime}\right)=\sum_{\beta_{k}} \operatorname{Tr}\left(\cdots P_{\alpha_{k}}^{k} \otimes R_{\beta_{k}}^{k} \cdots \rho \cdots P_{\alpha_{k}^{\prime}}^{k} \otimes R_{\beta_{k}}^{k} \cdots\right)
$$

Finally, the assumed correlated between the alternative $\alpha_{k}$ in $A$ and $\beta_{k}$ in $B$ means that the terms of the form $P_{\alpha_{k}}^{k} \otimes R_{\beta_{k}}^{k}$ on each side will yield zero when operating on everything that came earlier in the chain, unless $\alpha_{k}=\beta_{k}$. Eq.(3.5) will therefore be diagonal in $\alpha_{k}$. Repeating the argument for all other values of $k$, we thus find that, as advertized, a perfect and persistent correlation of alternatives of $A$ with those of $B$ leads to exact decoherence of the histories of $A$. It is not just the consistency condition (2.6) that is satisfied through persistent correlation with another subsystem, but the stronger condition of decoherence, (3.1). This argument was inspired by an argument given by Hartle [14] in his discussion of the recovery of the Copenhagen interpretation from the decoherent histories approach. A more detailed version of it is given in Ref.[32].

\subsection{Decoherence Implies Generalized Records}

There is a converse to the above result, namely that Eq.(3.1), in a certain sense, implies the existence of records [12]. Consider the decoherence functional (2.5), for 
any system (not just the special one discussed above). Introduce the convenient notation

$$
C_{\underline{\alpha}}=P_{\alpha_{n}}\left(t_{n}\right) \cdots P_{\alpha_{1}}\left(t_{1}\right)
$$

Let the initial state be pure, $\rho=|\Psi\rangle\langle\Psi|$. In this case, the decoherence condition (3.1) is referred to as medium decoherence. It implies that the states $C_{\underline{\alpha}}|\Psi\rangle$ are an orthogonal (but in general incomplete) set. There therefore exists a set of projection operators $R_{\beta}$ (not in general unique) of which these states are eigenstates,

$$
R_{\underline{\beta}} C_{\underline{\alpha}}|\Psi\rangle=\delta_{\underline{\alpha} \underline{\beta}} C_{\underline{\alpha}}|\Psi\rangle
$$

Note that the $C_{\underline{\alpha}}$ 's are not themselves projectors in general. One may then consider histories consisting of the string of projections (3.9), adjoined by the projections $R_{\underline{\beta}}$ at any time after the final time. The decoherence functional for such histories is

$$
D\left(\underline{\alpha}, \underline{\beta} \mid \underline{\alpha}^{\prime}, \underline{\beta}^{\prime}\right)=\operatorname{Tr}\left(R_{\underline{\beta}} C_{\underline{\alpha}}|\Psi\rangle\langle\Psi| C_{\underline{\alpha}^{\prime}}^{\dagger} R_{\underline{\beta}^{\prime}}\right)
$$

These extended histories decohere exactly by virtue of (3.10) and (3.1), and thus the diagonal elements of $(3.11)$, which we denote $p(\underline{\alpha}, \underline{\beta})$, are true probabilities. The correlations contained in these probabilities may therefore be discussed. Indeed, Eq.(3.10) implies that $p(\underline{\alpha}, \underline{\beta})=\delta_{\underline{\alpha}} \underline{\beta} p(\underline{\alpha})$, and thus $\underline{\alpha}$ and $\underline{\beta}$ are perfectly correlated.

Medium decoherence therefore implies the existence of a string of alternatives $\beta_{1} \cdots \beta_{n}$, at some fixed moment of time after $t_{n}$, perfectly correlated with the string $\alpha_{1}, \cdots \alpha_{n}$ at the sequence of times $t_{1} \cdots t_{n}$. For this reason the projection operators $R_{\underline{\alpha}}$ are referred to as generalized records: information about the histories characterized by alternatives $\alpha_{1} \cdots \alpha_{n}$ is recorded somewhere. It is, however, not possible to say that the information resides in a particular subsystem, since we have not specified the form of the system $S$; indeed, it is generally not possible to divide it into subsystems.

\section{Towards a Quasiclassical Domain}

Given the framework sketched above, one of the principle aims of the decoherent histories approach is to demonstrate the emergence of an approximately classical world from an underlying quantum one, together with the quantum fluctuations 
about it described by the familiar Copenhagen quantum mechanics of measured subsystems. Such a state of affairs is referred to as a quasiclassical domain $[11,12,13]$. In more technical terms, a quasiclassical domain consists of a decoherent set of histories, characterized largely by the same types of variables at different times, and whose probabilities are peaked about deterministic evolution equations for the variables characterizing the histories.

The histories should, moreover, be maximally refined with respect to a specified degree of approximate decoherence. That is, one specifies a decoherence factor $\epsilon$ in the approximate decoherence condition discussed above. This should, for example, be chosen so that the probabilities are defined to a precision far beyond any conceivable test. Then, the histories should be fine-grained (e.g., by reducing the widths of the projections) to the point that further fine-graining would lead to violation of the specified degree of approximate decoherence. The resulting set of histories are then called maximally refined. The reason for maximally refining the histories is to reduce as much as possible any apparent subjective element in the choice of coarse-graining.

Given the Hamiltonian and initial state of the system, one's task is to compute the decoherence functional for various different choices of histories, and see which ones lead to quasiclassical behaviour. As suggested by the discussion at the end of Section 2, there could be - and probably are - many such sets of variables leading to quasiclassical behaviour. An important problem is to find as many such sets as possible and develop criteria to distinguish between them. One useful criterion is whether a quasiclassical domain can support the existence of an information gathering and utilizing system, or IGUS. This is a complex adaptive system that exploits the regularities in its environment in such a way as to ensure its own survival. This particular criterion may rule out domains described by particularly bizarre decoherent sets of histories, such as ones described by completely different variables at each moment of time, because the IGUS may not have sufficient information processing capabilities to assimilate its environment. Also, criteria such as the existence of IGUSes alleviate to some degree the multiplicity of consistent sets of histories dis- 


\subsection{Histories of Hydrodynamic Variables}

What are the sets of variables that can lead to quasiclassical behaviour? One particular set of variables that are strong candidates for it are the integrals over small volumes of locally conserved densities. A generic system will usually not have a natural separation into "system" and "environment", and it is one of the strengths of the decoherent histories approach that it does not rely on such a separation. Certain variables will, however, be distinguished by the existence conservation laws for total energy, momentum, charge, particle number, etc. Associated with such conservation laws are local conservation laws of the form

$$
\frac{\partial \rho}{\partial t}+\nabla \cdot \mathbf{j}=0
$$

The candidate quasiclassical variables are then

$$
Q_{V}=\int_{V} d^{3} x \rho(\mathbf{x})
$$

If the volume $V$ over which the local densities are smeared is infinite, $Q_{V}$ will be an exactly conserved quantity. In quantum mechanics it will commute with the Hamiltonian, and, as is easily seen, histories of $Q_{V}$ 's will decohere exactly. If the volume is finite but large compared to the microscopic scale, $Q_{V}$ will be slowly varying compared to all other dynamical variables. This is because the local conservation law (4.1) permits $Q_{V}$ to change only by redistribution, which is limited by the rate at which the locally conserved quantity can flow out of the volume. Because these quantities are slowly varying, histories of them should approximately decohere. Furthermore, the fact that the $Q_{V}$ 's are slowly varying may also be used, at least classically, to derive an approximately closed set of equations involving only those quantities singled out by the conservation laws. These equations are, for example, the Navier-Stokes equations, and the derivation of them is a standard (although generally non-trivial) exercise in non-equilibrium statistical mechanics [36]. One of the current goals of the decoherent histories approach is to reexpress this derivativion in the language of histories [37].

\subsection{Quantum Brownian Motion Models}

Many concrete investigations of the mechanics of decoherence have actually 
concerned quantum Brownian motion models, primarily because calculations can be carried out with comparative ease $[12,31]$. These have proved to be quite instructive. Very briefly, such models consist of a particle of mass $M$ in a potential $V(x)$ linearly coupled to an environment consisting of a large bath of harmonic oscillators in a thermal state at temperature $T$, and characterized by a dissipation coefficient $\gamma$. The types of histories commonly considered are sequences of approximate positions of the Brownian particle, specified up to some width $\sigma$, whilst the environment of oscillators is traced over.

The results may briefly be summarized as follows. Decoherence through interaction with the environment is an extremely effective process. For example, for a particle whose macroscopic parameters (mass, timescale, etc.) are of order 1 in c.g.s. units, and for an environment at room temperature, the degree of approximate decoherence is of order $\exp \left(-10^{40}\right)$, a very small number. The probabilities for histories of positions are then strongly peaked about the classical equations of motion, but modified by a dissipation term,

$$
M \ddot{x}+M \gamma \dot{x}+V^{\prime}(x)=0
$$

There are fluctuations about classical predictability, consisting of the ubiquitous quantum fluctuations, adjoined by thermal fluctuations from the interaction with the environment. There is a generally a tension between the demands of decoherence and classical predictability, due to the fact that the degree of decoherence improves with increasing environment temperature, but predictability deteriorates, because the fluctuations about (4.3) grow. However, if the particle is sufficiently massive, it can resist the thermal fluctuations and a compromise regime can be found in which there is a reasonable degree of both decoherence and classical predictability.

\section{Decoherent Histories and Quantum State Diffusion}

The decoherent histories approach is closely connected to the quantum state diffusion (QSD) approach to open systems. In that approach, the master equation for the reduced density operator of an open system (essentially a closed system in which one focuses on a particular subsystem) is solved by exploiting a purely mathematical connection with a certain non-linear stochastic Schrödinger equation 
(Ito equation) [38]. Solutions to the Ito equation turn out to correspond rather closely to the results of actual laboratory experiments (e.g., in quantum optics), and are therefore held to describe individual systems and processes. For example, in a quantum Brownian motion model, the solutions to the Ito equation become localized about points in phase space following the classical equations of motion. The connection with the decoherent histories approach is that, loosely speaking, the solutions to the Ito equation may be thought of as the individual histories belonging to a decoherent set [39]. More precisely, the variables that localize in the QSD approach also define a decoherent set of histories in the decoherent histories approach. The degrees of localization and of decoherence are related, and the probabilities assigned to histories in each case are essentially the same. This connection could be a very useful one, both conceptually and computationally, and efforts to exploit it are being made.

\section{What Have We Gained?}

In this contribution I have tried to give a brief overview of the decoherent histories approach to quantum theory. What has the decoherent histories approach taught us?

At the level of ordinary quantum mechanics, applied to laboratory situations, two things have been gained. First of all, a minimal view of the decoherent histories approach is that it is in a sense a more refined version of the Copenhagen interpretation. It rests on a considerable smaller number of axioms, and in particular, it is a predictive formulation of quantum mechanics that does not rely on any kind of assumptions referring to measurement or to a classical domain. It is internally consistent and reproduces all the experimental predictions of the Copenhagen approach. Secondly, it provides a clear set of criteria for the application of ordinary logic in quantum mechanics. Since many of the conceptual difficulties of quantum mechanics are essentially logical ones, e.g., the EPR paradox, a clarification of the applicability of logic has been argued to lead to their resolution [7,21,24]. Such a resolution is not strictly possible in Copenhagen quantum mechanics, because it does not offer clear guidelines for the application of ordinary logic.

There will, of course, always be some who claim that they can finesse their way 
through any difficulty of quantum mechanics without having to worry about the somewhat cumbersome machinery of the histories approach described here. In this connection, Omnès has to say the following [26]:

"It may be true, as some people say, that everything is in Bohr, but this has been a matter for hermeneutics, with the endless disputes any scripture will lead to. It may also happen that he guessed the right answers, but the pedagogical means and the necessary technique details were not yet available to him. Science cannot, however, proceed by quotations, however elevated the source. It proceeds by elucidation, so that feats of genius can become ordinary learning for beginners."

Intuition alone may be sufficient to see some through the difficulties of nonrelativistic quantum mechanics, but if we are to extend quantum theory to the entire universe, a reliable vehicle for travel beyond the domain of our intuition is required. For quantum cosmology, the development of the decoherent histories approach has been a considerable bonus. The decoherent histories approach supplies an unambiguous, workable and predictive scheme for actually applying quantum mechanics to genuinely closed systems. Furthermore, as discussed at some length in this paper, it supplies a conceptually clear method of discussing the emergence of classicality in closed quantum systems, and this is perhaps its greatest success.

Still outstanding are the largely technical difficulties of quantum cosmology connected with quantizing gravity. However, it is possible that the histories approach might be of use there also. The focus on histories may circumvent the "problem of time" encountered in most canonical approaches to quantum gravity. Isham and collaborators, for example, are currently exploring the possibility of histories-based formulations of quantum theory that do not rely on the conventional Hilbert space structure, or on the existence of a preferred time coordinate $[40,41,42]$, building on an earlier suggestion of Hartle [16]. Much remains to be done, but on both conceptual and technical grounds, the histories approach to quantum cosmology appears to be a particularly promising avenue for future research.

Further aspects of the decoherent histories approach are discussed in 


\section{Acknowledgments}

I am grateful to the organizers for giving me the opportunity to take part in such an interesting meeting. I would also like to thank numerous colleagues for useful conversations, especially Lajos Diósi, Fay Dowker, Murray Gell-Mann, Nicolas Gisin, Jim Hartle, Chris Isham, Adrian Kent, Seth Lloyd, Roland Omnès, Ian Percival, Trevor Samols, Dieter Zeh and Wojciech Zurek. This work was supported by a University Research Fellowship from the Royal Society.

\section{References}

1. A useful source of literature on the Copenhagen interpretation is, J. A. Wheeler and W. Zurek (eds.), Quantum Theory and Measurement (Princeton University Press, Princeton, NJ, 1983).

2. A. J. Leggett, Suppl.Prog.Theor.Phys. 69, 80 (1980). Macroscopic Quantum Systems and the Quantum Theory of Measurement.

3. J. J. Halliwell, in General Relativity and Gravitation 1992, edited by R. J. Gleiser, C. N. Kozameh and O. M. Moreschi (IOP Publishers, Bristol, 1993). The Interpretation of Quantum Cosmological Models.

4. J. A. Wheeler, in Relativity, Groups and Topology, eds. C.DeWitt and B.DeWitt (Gordon and Breach, New York, 1963).

5. R. B. Griffiths, J.Stat.Phys. 36, 219 (1984). Consistent Histories and the Interpretation of Quantum Mechanics.

6. R. B. Griffiths, Phys.Rev.Lett. 70, 2201 (1993). Consistent Interpretations of Quantum Mechanics Using Quantum Trajectories.

7. R. B. Griffiths, Am.J.Phys. 55, 11 (1987). Correlations in Separated Quantum Systems: A Consistent History Analysis of the EPR Problem.

8. R. B. Griffiths, Pittsburgh preprint (1994). A Consistent History Approach to the Logic of Quantum Mechanics. 
9. R. Griffiths, Pittsburgh preprint (1994). Consistent Quantum Counterfactuals.

10. M. Gell-Mann, The Quark and the Jaguar (Little, Brown and Company, London, 1994).

11. M. Gell-Mann and J. B. Hartle, in Complexity, Entropy and the Physics of Information, SFI Studies in the Sciences of Complexity, Vol. VIII, W. Zurek (ed.) (Addison Wesley, Reading, 1990); and in Proceedings of the Third International Symposium on the Foundations of Quantum Mechanics in the Light of New Technology, S. Kobayashi, H. Ezawa, Y. Murayama and S. Nomura (eds.) (Physical Society of Japan, Tokyo, 1990). Quantum Mechanics in the Light of Quantum Cosmology.

12. M. Gell-Mann and J. B. Hartle, Phys.Rev. D47, 3345 (1993). Classical Equations for Quantum Systems.

13. M. Gell-Mann and J. B. Hartle, Santa Barbara preprint (1994). Equivalent Sets of Histories and Multiple Quasiclassical Domains.

14. J. B. Hartle, in Quantum Cosmology and Baby Universes, S. Coleman, J. Hartle, T. Piran and S. Weinberg (eds.) (World Scientific, Singapore, 1991). The Quantum Mechanics of Cosmology.

15. J. B. Hartle, Phys.Rev. D44, 3173 (1991). Spacetime Coarse-Grainings in Non-Relativistic Quantum Mechanics.

16. J. B. Hartle, in, Proceedings of the 1992 Les Houches Summer School, Gravitation et Quantifications, B.Julia (ed.). Spacetime Quantum Mechanics and the Quantum Mechanics of Spacetime.

17. J. B. Hartle, in the festschrift for C. Misner, edited by B.L. Hu, M. P. Ryan amd C. V. Vishveshwara (Cambridge University Press, Cambridge, 1993). The Quantum Mechanics of Closed Systems.

18. J. B. Hartle, in the festschrift for D. Brill, edited by B.L. Hu and T. Jacobson (Cambridge University Press, Cambridge, 1993). The Reduction of the State Vector and Limitations on Measurement in the Quantum Mechanics of Closed Systems.

19. J. B. Hartle, Santa Barbara preprint (1994), to appear in Proceedings of the Lanczos Centenary Meeting. Quasiclassical Domains in a Quantum Universe. 
20. R. Omnès, J.Stat.Phys. 53, 893 (1988). Logical Reformulation of Quantum Mechanics. I. Foundations.

21. R. Omnès, J.Stat.Phys. 53, 933 (1988). Logical Reformulation of Quantum Mechanics. II. Interferences and the Einstein-Podolsky-Rosen Experiment.

22. R. Omnès, J.Stat.Phys. 53, 957 (1988). Logical Reformulation of Quantum Mechanics. III. Classical Limit and Irreversibility.

23. R. Omnès, J.Stat.Phys. 57, 357 (1989). Logical Reformulation of Quantum Mechanics. IV. Projectors in Semiclassical Physics.

24. R. Omnès, Phys.Lett. A138, 157 (1989). The Einstein-Podolsky-Rosen Problem: A New Solution.

25. R. Omnès, Ann.Phys. 201, 354 (1990). From Hilbert Space to Common Sense: A Synthesis of Recent Progress in the Interpretation of Quantum Mechanics.

26. R. Omnès, Rev.Mod.Phys. 64, 339 (1992). Consistent Intepretations of Quantum Mechanics.

27. R. Omnès, Phys.Lett. A187, 26 (1994). A Model for the Uniqueness of Data and Decoherent Histories.

28. R. Omnès. J.Stat.Phys. 62, 841 (1991). About the Notion of Truth in Quantum Mechanics.

29. B. d'Espagnat, J.Stat.Phys. 56, 747 (1989). Are There Realistically Interpretable Local Theories?

30. H. F. Dowker and A. Kent, Newton Institute preprint NI-94006 (1994). On the Consistent Histories Formulation of Quantum Mechanics.

31. H. F. Dowker and J. J. Halliwell, Phys. Rev. D46, 1580 (1992). Quantum Mechanics of History: The Decoherence Functional in Quantum Mechanics.

32. J. J. Halliwell, in Stochastic Evolution of Quantum States in Open Systems and Measurement Processes, edited by L. Diósi (World Scientifc, Singapore, 1994). Aspects of the Decoherent Histories Approach to Quantum Mechanics

33. S. Saunders, Harvard preprint (1992). The Quantum Block Universe.

34. S. Saunders, Phys.Lett. A184, 1 (1993). Decoherence and Evolutionary Adaptation. 
35. S. Saunders, Harvard preprint (1993). Decoherence, Relative States and Evolutionary Adaptation.

36. D. Forster, Hydrodynamic Fluctuations, Broken Symmetry and Correlation Functions (Benjamin, Reading, MA, 1975).

37. J. J. Halliwell and J. B. Hartle, work in progress.

38. N. Gisin and I.C. Percival, J.Phys. A25, 5677 (1992). The Quantum State Diffusion Model Applied to Open Systems.

39. L. Diósi, N. Gisin, J. J. Halliwell and I.C.Percival, Imperial College preprint IC 93-94/25 (1994). Decoherent Histories and Quantum State Diffusion.

40. C. Isham, Imperial Preprint IC/92-93/39 (1993). Quantum Logic and the Histories Approach to Quantum Theory.

41. C. Isham and N. Linden, Imperial College preprint IC/TP/93-94/35 (1994). Quantum Temporal Logic and Decoherence Functionals in the Histories Approach to Generalized Quantum Theory.

42. C. Isham, N. Linden and S.Schreckenberg, Imperial College preprint IC/TP/9394/42 (1994). The Classification of Decoherence Functionals: An Analogue of Gleason's Theorem.

43. A. Albrecht, Phys.Rev. D46, 5504 (1092). Investigating Decoherence in a Simple System.

44. A. Albrecht, Imperial Preprint 92-93/03 (1992). Following a Collapsing Wave Function.

45. M. Blencowe, Ann.Phys. 211, 87 (1991). The Consistent Histories Interpretation of Quantum Fields in Curved Spacetime.

46. T. Brun, Phys. Rev. D47, 3383 (1993). Quasiclassical Equations of Motion for Nonlinear Brownian Systems.

47. T. Brun, Caltech preprint (1994). The Decoherence of Phase Space Histories.

48. E. Calzetta and B. L. Hu, in Directions in General Relativity, edited by B. L. Hu and T. A. Jacobson (Cambridge University Press, Cambridge, 1993). Decoherence of Correlation Histories. 
49. L. Diósi, Budapest preprint KFKI-1992-03/A (1992). Coarse Graining and Decoherence Translated into von Neumann Language.

50. L. Diósi, Budapest preprint KFKI-RMKI-23 (1993). Unique Family of Consistent Histories in the Caldeira-Leggett Model.

51. L. Diósi, Budapest preprint KFKI-RMKI-25 (1993). Comment on, "Consistent Intepretation of Quantum Mechanics Using Quantum Trajectories".

52. L. Diósi, Budapest preprint KFKI-RMKI-28 (1993). Unique Quantum Paths by Continuous Diagonalization of the Density Operator.

53. J. Finkelstein, San José preprint SJSU/TP-93-10 (1993). On the Definition of Decoherence.

54. S. Goldstein and D. N. Page, University of Alberta preprint Alberta-Thy-43-93, gr-qc 9403055 (1993). Linearly Positive Histories.

55. J. J. Halliwell, Phys.Rev. D48, 2739 (1993). Quantum Mechanical Histories and the Uncertainty Principle: Information-Theoretic Inequalities.

56. J. J. Halliwell, Phys.Rev. D48, 4785 (1993). Quantum Mechanical Histories and the Uncertainty Principle: II. Fluctuations about Classical Predictability.

57. R. Laflamme and A. Matacz, Los Alamos preprint (1993). Decoherence Functional and Inhomogeneities in the Early Universe.

58. Y. Ohkuwa, Santa Barbara preprint, UCSBTH-92-40 (1992). Decoherence Functional and Probability Interpretation.

59. J. P. Paz and W. H. Zurek, Phys.Rev. D48, 2728 (1993). Environment-Induced Decoherence, Classicality and Consistency of Quantum Histories.

60. R. Sorkin, Syracuse preprint SU-GP-93-12-1 (1993). Quantum Mechanics as Quantum Measure Theory.

61. J. Twamley, Adelaide preprint ADP-93-202/M16 (1993). Inconsistency Between Alternative Approaches to Quantum Decoherence in Special Systems.

62. J. Twamley, Adelaide preprint ADP-93-208/M19 (1993). Phase Space Decoherence: A Comparison between Consistent Histories and Environment-Induced Superselection. 
63. W. Zurek, in Physical Origins of Time Asymmetry, edited by J. J. Halliwell, J. Perez-Mercader and W. Zurek (Cambridge University Press, Cambridge, 1994). Preferred Sets of States, Predictability, Classicality and Environment-Induced Decoherence. 